\title{
Photoacoustic Doppler Effect from Flowing Small Light-Absorbing Particles
}

\author{
Hui Fang, Konstantin Maslov, and Lihong V. Wang* \\ Optical Imaging Laboratory, Department of Biomedical Engineering, Washington University in St. Louis, \\ One Brookings Dr., St. Louis, Missouri 63130, USA
}

(Received 22 July 2007; published 29 October 2007)

\begin{abstract}
From the flow of a suspension of micrometer-scale carbon particles, the photoacoustic Doppler shift is observed. As predicted theoretically, the observed Doppler shift equals half of that in Doppler ultrasound and does not depend on the direction of laser illumination. This new physical phenomenon provides a basis for developing photoacoustic Doppler flowmetry, which can potentially be used for detecting fluid flow in optically scattering media and especially low-speed blood flow of relatively deep microcirculation in biological tissue.
\end{abstract}

DOI: 10.1103/PhysRevLett.99.184501

Laser flowmetry and acoustic flowmetry based on the Doppler effect [1] have become valuable tools for fluid mechanics measurements [2]. More recently, lowcoherence optical Doppler tomography [3] was developed. They all require the presence of small scattering tracer particles to provide detectable backscattering signals. In measurement of tissue blood flow [4,5], red blood cells work as endogenous scattering tracer particles.

In this Letter, however, we describe the observation of a new physical phenomenon-the photoacoustic Doppler (PAD) effect from moving particles - and its application for flow measurement. Here, small light-absorbing particles [6] are used as tracer particles. The term "photoacoustics" [7] (or the equivalent "optoacoustics" [8]) usually refers to the generation of acoustic waves by modulated or pulsed optical radiation, an effect discovered by A. G. Bell in 1880 . The PAD effect thus deals with the Doppler frequency shift of the generated acoustic waves. Previously, the PAD effect due to moving light excitation was discussed in the research of a moving thermoacoustic array $[9,10]$. In those studies, no flow in the medium was involved. Instead, a laser beam was scanned over an absorbing liquid. As a result, the Doppler shift was found to depend on the laser scanning speed.

Compared to the aforementioned conventional scattering-based Doppler flowmetry, PAD-based flowmetry should have much lower background noise. If there are no other absorbers besides the tracer particles inside the measured volume, the PAD signal should only come from the tracer particles. PAD flowmetry can potentially quantify tissue blood flow noninvasively because red blood cells are dominant endogenous light-absorbing tracer particles that can absorb light 100 times more than the background [11]. In contrast, the scattering-based Doppler signal usually suffers from overwhelming background reflection from the flow's surrounding medium [12-14].

Let us first consider theoretically the PAD shift from a flowing light-absorbing particle. The particle is suspended in a liquid and flows with the liquid along velocity vector $\vec{V}$ (Fig. 1). When the particle is illuminated by an amplitude-
PACS numbers: 47.80.Cb, 47.35.Rs, 47.55.Kf, 87.80.-y

modulated continuous-wave laser beam, an acoustic wave is generated due to the photoacoustic effect, and the acoustic wave can be detected by an ultrasonic transducer. If the laser beam is modulated at frequency $f_{O}$ with $100 \%$ modulation depth, its intensity $I$ as a function of time $t$ can be express as

$$
I=I_{0}\left[1+\cos \left(2 \pi f_{O} t\right)\right] / 2,
$$

where $I_{0}$ denotes the peak intensity. Such a laser beam can be treated as a photon density wave with frequency $f_{O}$. If the particle is not in motion, the photoacoustic wave has the same frequency as $f_{O}[15,16]$. Otherwise, the photoacoustic wave is subject to a Doppler shift.

Because photoacoustic pressure amplitude is proportional to the absorbed optical power density, the Doppler shift depends on the frequency of the intensity $f_{O}$ instead of the frequency of the field. The Doppler shift should also depend on the flow velocity $V$ and the flow direction angles $\alpha$ and $\theta$ as illustrated in Fig. 1 , where $\vec{K}_{O}$ and $\vec{K}_{A}$ refer to the wave vectors of the photon density and acoustic waves, respectively. If $V$ is much less than the sound speed, the Doppler shift can be expressed as $-f_{O} \frac{V}{c_{O}} \cos \alpha+f_{O} \frac{V}{c_{A}} \times$ $\cos \theta$, where $c_{O}$ and $c_{A}$ denote the speeds of light and

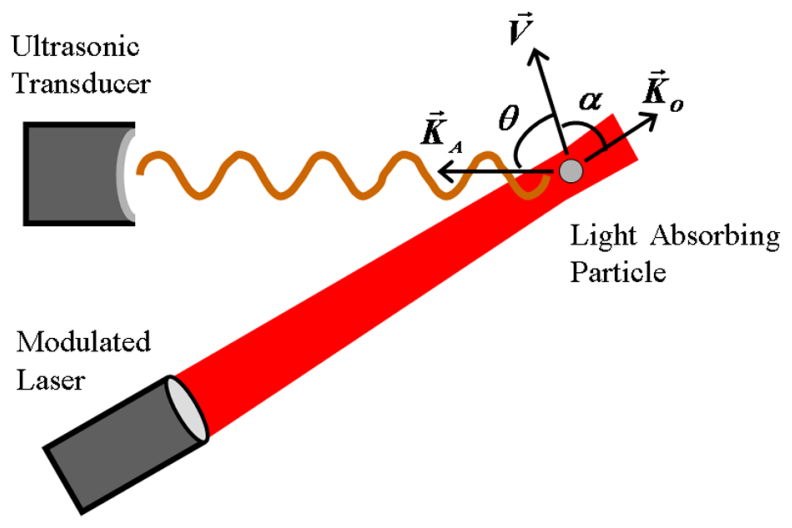

FIG. 1 (color online). Schematic for photoacoustic Doppler shift. The small light-absorbing particle moving along velocity vector is illuminated by modulated continuous-wave light. 
sound, respectively, in the medium. In this expression, the first term represents the shift in the frequency of the photon density wave "seen" by the particle as a moving receiver; the second term represents the shift in the frequency of the photoacoustic wave "observed" by the ultrasonic transducer, where the particle works as a moving source. Because $c_{O} / c_{A} \sim 10^{5}$ and $V \ll c_{A}$, usually only the second term is detectable. Therefore, we have

$$
f_{\mathrm{PAD}}=f_{O} \frac{V}{c_{A}} \cos \theta
$$

This shift equals half of the shift in pulse-echo Doppler ultrasound and does not depend on the direction of the laser illumination. Unlike the laser Doppler effect, the PAD effect deals with a photon density wave instead of an optical wave, and the photon density wave has a much longer wavelength than the acoustic wave. Therefore, the Doppler shift of the illumination laser is negligible.

The experimental setup (Fig. 2) we used is based on continuous-wave (cw) photoacoustic microscopy $[17,18]$. Briefly, a cw diode laser (center wavelength: $784 \mathrm{~nm}$; average power: $120 \mathrm{~mW}$ ) was amplitude-modulated by a function generator ("function generator 1") with $100 \%$ modulation depth at frequency $f_{O}=2.4550 \mathrm{MHz}$. The laser beam was focused onto the flow sample with a spot of $\sim 1.0 \mathrm{~mm}$ in diameter. A narrow band piezoelectric transducer (center frequency: $2.4550 \mathrm{MHz}$; numerical aperture NA: 0.85) was aligned to be confocal with the laser focal spot. The acoustic signal detected by the transducer was amplified by a narrow band preamplifier and relayed to the "signal" input of a lock-in detector. Another function generator ("function generator 2") was synchronized with "function generator 1" to provide a "reference" input for

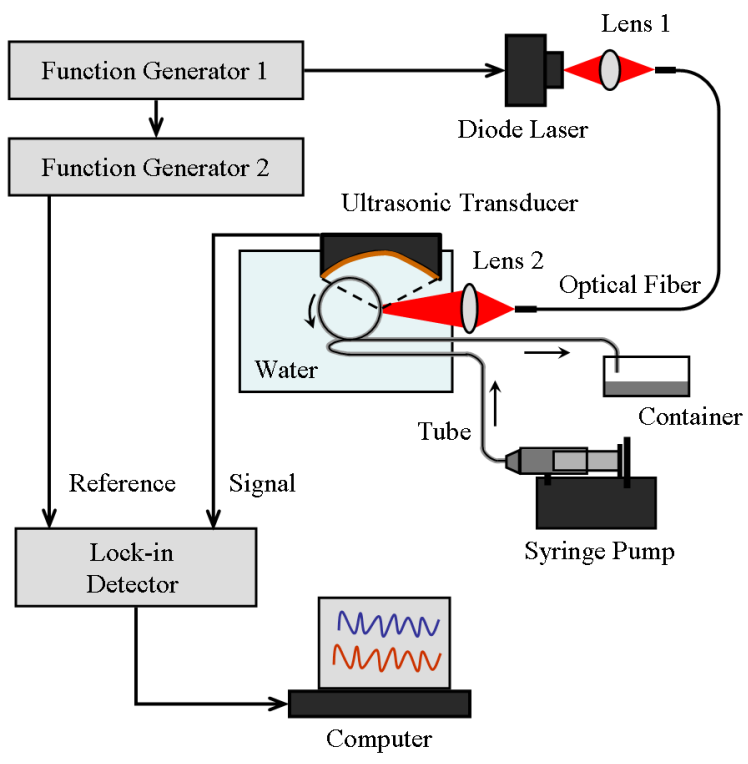

FIG. 2 (color online). Diagram of the experimental setup for continuous-wave photoacoustic Doppler flow measurement. the lock-in detector with frequency $f_{\text {Ref }}=f_{O}$. The lock-in detector uses phase sensitive detection to produce a pair of quadrature-demodulated outputs " $X$ " and " $Y$," where " $X$ " is the low-pass filtered product of the signal and the reference, and " $Y$ " is the low-pass filtered product of the signal and the $+90^{\circ}$ phase shifted reference. The bandwidth of the low-pass filter is determined by the time constant and was chosen to be $102 \mathrm{~Hz}$ in the experiment (the time constant was set as $1 \mathrm{~ms}$ ). The lock-in detector worked at the maximum sampling rate of $512 \mathrm{~Hz}$ with a total sampling points of 15360 (the maximum is 16800). The digitized " $X$ " and " $Y$ " were then transferred to the computer and analyzed spectrally. The frequency difference between the signal and the reference is the Doppler shift of the signal.

As shown in Fig. 2, the fluid flow was generated by a syringe pump (BSP-99M, Braintree Scientific) with a $10 \mathrm{cc}$ syringe (Multifit, Becton, Dickinson \& Co.) and a Tygon@ tube (inside diameter: $0.51 \mathrm{~mm}$; outside diameter: 1.53 mm; S-54-HL, Saint-Gobain Performance Plastics). The tube was formed into a circle and mechanically fixed inside a water tank. A vertical segment of the circle was measured at a downstream distance of $\sim 50 \mathrm{~cm}$ from the flow entry connected to the syringe. Through the syringe pump, the volume flow rate, $Q$, could be manually set from 0.04 to $39.6 \mathrm{cc} / \mathrm{hr}$ with steps of $0.04 \mathrm{cc} / \mathrm{hr}$.

The flow sample was a particle suspension with volume fraction $\phi \approx 15 \%$, where the particle diameters distributed from 2 to 12 micrometers (carbon glassy spherical powder, 484 164, Sigma-Aldrich). The solution for suspending the particles was made by dissolving an appropriate amount of solid sodium polytungstate (Sometu) into distilled water so that its mass density became about $1.46 \mathrm{~g} / \mathrm{cm}^{3}$, which matched that of the particles. Also, $1 \%$ volume of Tween-20 (Sigma-Aldrich) was added into the solution to reduce particle aggregation. The absorption coefficient of the suspension was estimated to be about $1.0 \mathrm{~mm}^{-1}$ by a transmission measurement using the same diode laser.

We first took a set of measurements to study the dependence of the PAD shift on the flow speed. The flow direction was set toward the transducer. Figure 3 plots the shifts versus the average flow velocity $\bar{V}_{S}$, which was calculated by dividing $Q$ by the cross sectional area of the tube. The square symbols indicate the mean Doppler shifts, and the error bars indicate the standard deviations, both calculated from the measured Doppler power spectra. As an example, the power spectrum for $\bar{V}_{S}=4.4 \mathrm{~mm} / \mathrm{s}$ is plotted as an inset in Fig. 3. For comparison with the theory, the mean shift is predicted from Eq. (2), i.e., $f_{O} \frac{\bar{V}_{S}}{c_{A}}\left(\frac{\cos 0^{\circ}+\cos 60^{\circ}}{2}\right)$, where $c_{A}=1500 \mathrm{~m} / \mathrm{s}$, and $0^{\circ}$ and $60^{\circ}$ represent the detection angular range of the ultrasonic transducer due to its numerical aperture. As can be seen, the measured mean Doppler shifts agree well with the theoretical predictions, whereas each standard deviation is about half of the associated mean shift. We repeated the same set of measure- 


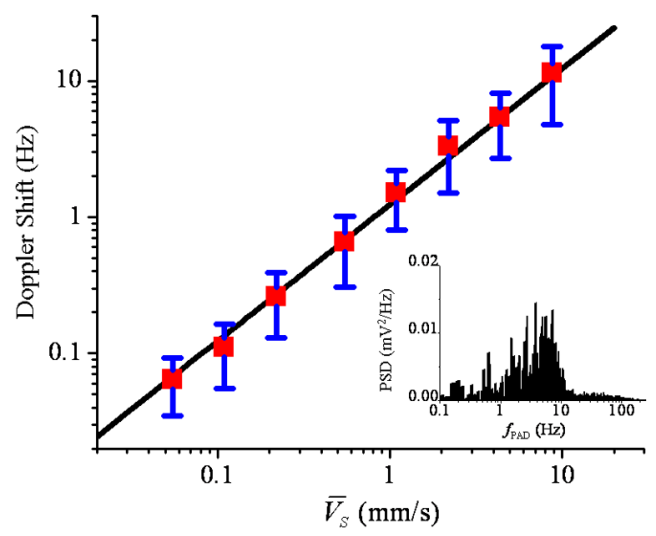

FIG. 3 (color online). Experimentally measured photoacoustic Doppler frequency shift as a function of average flow velocity. Square symbol: mean frequency shift; Error bar: standard deviation of the shift; solid line: theoretically predicted mean shift from Eq. (2). The inset plots the power spectrum for the average flow velocity of $4.4 \mathrm{~mm} / \mathrm{s}$. PSD: power spectral density.

ments 10 times, and the results were consistent with those shown in Fig. 3.

The observed standard deviations were modeled by taking into account the flow velocity profile, the flow angular distribution, and the transit time of each particle through the detection volume [19]. It was found that a parabolic velocity profile led to standard deviations greater than the mean shifts, whereas a fully blunted velocity profile (i.e., zero at the tube boundary and constant elsewhere) provided better agreement with the experimental observation. This finding suggested that the actual flow was blunted. Previous studies dealing with a similar type of flow also demonstrated that the velocity profile deviated from parabola and blunted to a shape that depended on parameters such as the particle volume concentration, particle size, flow channel size, flow speed, and downstream distance [20,21].

As also shown in Fig. 3, the measurable $\bar{V}_{S}$ was in the range of $0.055-8.8 \mathrm{~mm} / \mathrm{s}$. The maximum measurable $\bar{V}_{S}$ was limited by the signal-to-noise ratio. When $\bar{V}_{S}$ increased, the power spectrum broadened linearly with $\bar{V}_{S}$. At the same time, the spectral amplitude decreased and eventually approached the noise level. Increasing the laser power or improving the transducer sensitivity can extend the maximum. In contrast, the minimum measurable $\bar{V}_{S}$, which represents the velocity sensitivity of the system, was limited by the frequency resolution of the system $(0.033 \mathrm{~Hz})$. Increasing the number of sampling points can improve the minimum. The theoretical limit of the velocity sensitivity, estimated from the PAD broadening due to the Brownian motion of tracer particles, is $\frac{2 \pi f_{O}}{c_{A}} \cdot \frac{k_{B} T}{6 \pi \eta a} \approx 6.0 \times$ $10^{-7} \mathrm{~mm} / \mathrm{s}$, where the Boltzmann constant $k_{B}=1.38 \times$ $10^{-23} \mathrm{~J} / \mathrm{K}$, temperature $T=300 \mathrm{~K}$, viscosity coefficient $\eta=10^{-3} \mathrm{~Pa} \cdot \mathrm{s}$, and the average particle radius $a=$ $3.5 \mu \mathrm{m}$.
We then experimentally studied the dependence of the PAD shift on the flow direction. The flow direction was reversed by simply switching the two ends of the tube (one was connected with the syringe and the other was hung in the container as shown in Fig. 2). Figures 4(a) and 4(b) plot the ac components of the " $X$ " and " $Y$ " signals in a small time window for two flows with the same $\bar{V}_{S}=$ $2.20 \mathrm{~mm} / \mathrm{s}$ but opposite directions. For clarity, the dc offsets, which were different for " $X$ " and " $Y$ " (about $-3.5 \mathrm{mV}$ for " $X$ " and about $+2.0 \mathrm{mV}$ for " $Y$ "), were removed. As can be seen, the ac components of " $X$ " and " $Y$ " are similar in amplitude but different in phase with a shift of $\pi / 2$. However, " $Y$ " lags " $X$ " in Fig. 4(a), whereas " $Y$ " leads " $X$ " in Fig. 4(b).

This observation can be understood from the following simplified model. We assume that the Doppler shift is infinitely narrow and takes on the experimentally observed mean frequency $\bar{f}_{\mathrm{PAD}}$. The " $X$ " and " $Y$ " signals, representing the photoacoustic signals that are quadraturedemodulated by the lock-in detector, can be expressed as

$$
\begin{aligned}
X= & A_{O} \cos \psi_{O}+\bar{A}_{D} \cos \left(2 \pi \bar{f}_{\mathrm{PAD}} t+\psi_{D}\right)+E_{X} \\
Y= & A_{O} \cos \left(\psi_{O}-\pi / 2\right) \\
& +\bar{A}_{D} \cos \left(2 \pi \bar{f}_{\mathrm{PAD}} t+\psi_{D}-\pi / 2\right)+E_{Y} .
\end{aligned}
$$

Here, $A_{O}$ and $\psi_{O}$ denote the amplitude and initial phase, respectively, of the unshifted photoacoustic signal; $\bar{A}_{D}$ and $\psi_{D}$ denote the amplitude and initial phase, respectively, of the PAD-shifted photoacoustic signal; and $E_{X}$ and $E_{Y}$ denote the noises. The dc components in both " $X$ " and " $Y$ " should come from the particles near the tube wall that have zero flow velocity because photoacoustic signals
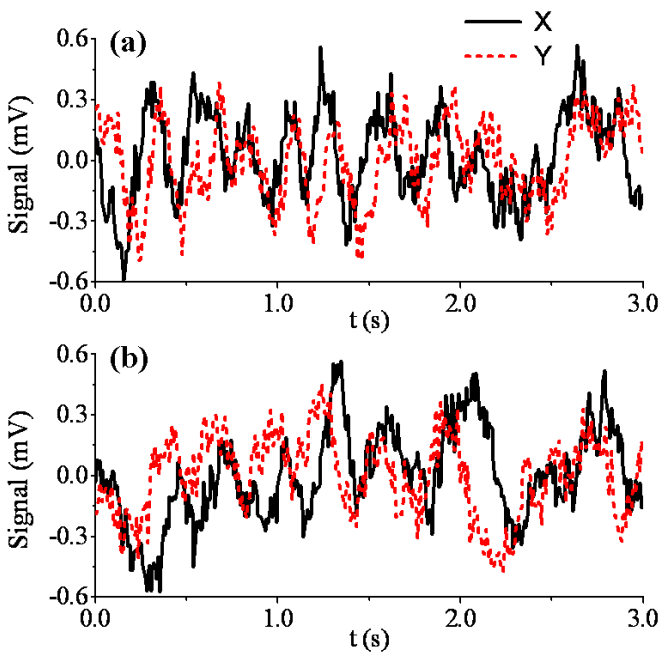

FIG. 4 (color online). Directional discrimination of flow in photoacoustic Doppler signals. Average flow velocity: $2.2 \mathrm{~mm} / \mathrm{s}$. (a) ac components of " $X$ " and " $Y$ " signals for a flow toward the transducer. (b) ac components of " $X$ " and " $Y$ " signals for a flow away from the transducer. 
come from only the particles. The two ac components are due to the PAD shift and have a phase difference of $\pi / 2$. For the flow measurement shown in Fig. 4(a), $\bar{f}_{\mathrm{PAD}}$ is positive; thus, "Y" lags "X." On the contrary, for the measurement shown in Fig. 4(b), $\bar{f}_{\mathrm{PAD}}$ is negative; thus, " $Y$ " leads " $X$." In addition, the dc components in " $X$ " and " $Y$ " can take on different values, whereas the ac components share the same amplitude.

In summary, we observed the PAD effect from micrometer-scale light-absorbing particles and used it to measure flow at an average flow velocity as low as $0.055 \mathrm{~mm} / \mathrm{s}$ and as high as $8.8 \mathrm{~mm} / \mathrm{s}$. We also observed the directional dependence of the PAD shift. As was discussed, the measurable range of average flow velocity can be extended in both directions through technical improvement.

Although the PAD flowmetry is in its infancy, our study showed its capability for measuring low-speed flow in a small channel. We expect it to be useful for measuring blood flow in microcirculation, which has average velocities from a fraction of $\mathrm{mm} / \mathrm{s}$ in capillaries to tens of $\mathrm{mm} / \mathrm{s}$ in small veins and arterials. The PAD flowmetry should be able to measure this type of blood flow at tissue depths beyond a few millimeters with preserved directional information, which is still a challenge with existing flowmetry techniques [22]. Acoustic flowmetry has difficulty measuring slow blood flow because tissue background scattering (so called "clutter" noise) swamps the Doppler signal at low frequency [13]. The PAD flowmetry has intrinsically low background and should have much less clutter noise. Although laser flowmetry and low-coherence optical Doppler tomography can measure microcirculation using the short optical wavelength, the flow directional information can get lost, and the detection depth is limited to about $1 \mathrm{~mm}$ because of multiple light scattering in tissue. The PAD flowmetry should be less hindered by multiple light scattering not only because the PAD shift does not involve the direction of laser illumination but also because photoacoustic imaging has demonstrated a greater imaging depth in tissue [23-25]. We are developing the PAD flowmetry for measuring blood flow of microcirculation in biological tissue.

We would like to thank Geng $\mathrm{Ku}$ for experimental assistance and thank Erich Stein and Roger Zemp for useful discussion. This project is supported by National Institutes of Health Grants Nos. R01 EB000712 and R01 NS046214.
*Corresponding author: lhwang@biomed.wustl.edu

[1] D. N. White, Ultrasound in Med. \& Biol. 8, 583 (1982).

[2] Fluid Mechanics Measurements, edited by Richard J. Goldstein (Taylor \& Francis, Bristol, 1996).

[3] Z.P. Chen, Y.H. Zhao, S.M. Srinivas, J.S. Nelson, N. Prakash, and R.D. Frostig, IEEE J. Sel. Top. Quantum Electron. 5, 1134 (1999).

[4] Laser-Doppler Blood Flowmetry, edited by A. P. Shepherd and P. A. Oberg (Kluwer Academic Publishers, Norwell, 1990).

[5] Peter Atkinson and John P. Woodcock, Doppler Ultrasound and its use in Clinical Measurement (Academic Press, London, 1982).

[6] C.F. Bohren and D. R. Huffman, Absorption and Scattering of Light by Small Particles (Wiley, New York, 1983).

[7] Andrew C. Tam, Rev. Mod. Phys. 58, 381 (1986).

[8] V.E. Gusev and A. A. Karabutov, Laser Optoacoustics (American Institute of Physics, New York, 1993).

[9] Al. A. Kolomenskii, Sov. Phys. Acoust. 33, 526 (1987).

[10] Y. H. Berthelot and L. J. Busch-Vishniac, J. Acoust. Soc. Am. 81, 317 (1987).

[11] L. V. Wang and H.-i Wu, Biomedical Optics: Principle and Imaging (Wiley, Hoboken, 2007).

[12] M. G. D. Karlsson and K. Wardell, J Biomed. Opt. 10, 064002 (2005).

[13] A. Heimdal and H. Torp, IEEE Trans. Ultrason. Ferroelectr. Freq. Control 44, 873 (1997).

[14] H. Ren and X. D. Li, Opt. Express 14, 6103 (2006).

[15] M. I. Khan and G. J. Diebold, Ultrasonics 33, 265 (1995).

[16] G. J. Diebold, T. Sun, and M. I. Khan, Phys. Rev. Lett. 67, 3384 (1991).

[17] K. Maslov and L. V. Wang, Proc. SPIE-Int. Soc. Opt. Eng. 6437, 64370P (2007).

[18] Y. Fan, A. Mandelis, G. Spirou, I. A. Vitkin, and W. M. Whelan, Phys. Rev. E 72, 051908 (2005).

[19] W. R. Brody and J. D. Meindl, IEEE Trans. Biomed. Eng. bme-21, 183 (1974).

[20] P. R. Nott and J. F. Brady, J. Fluid Mech. 275, 157 (1994).

[21] C. J. Koh, P. Hookham, and L. G. Leal, J. Fluid Mech. 266, 1 (1994).

[22] T. Jansson, H. W. Persson, and K. Lindstrom, Proc. Inst. Mech. Eng., H J. Eng. Med. 213, 91 (1999).

[23] C. G. A. Hoelen, F. F. M. de Mul, R. Pongers, and A. Dekker, Opt. Lett. 23, 648 (1998).

[24] A. A. Oraevsky and A. A. Karabutov, in Biomedical Photonics Handbook, edited by Vol-Dinh (CRC Press, Boca Raton, 2003), Vol. PM125.

[25] H. F. Zhang, K. Maslov, and L. V. Wang, Nat. Biotechnol. 24, 848 (2006). 